onkologie aktuell

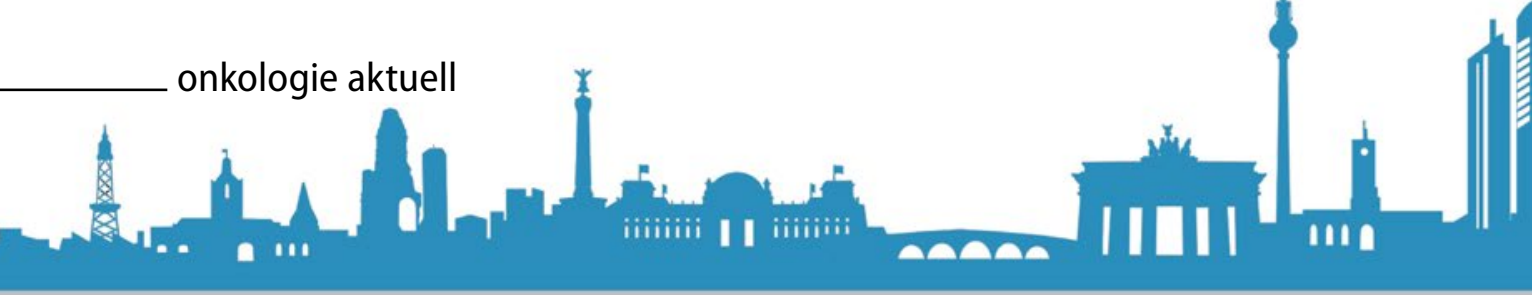

\title{
Neue Perspektiven in der Krebsmedizin
}

\author{
Der 33. Deutsche Krebskongress (DKK) steht bevor. Er ist der größte onkologische Fachkongress \\ im deutschsprachigen Raum und wird gemeinsam von der Deutschen Krebsgesellschaft und der \\ Deutschen Krebshilfe ausgerichtet.
}

\begin{abstract}
Unter dem Motto „Perspektiven verändern Krebs - Krebs verändert Perspektiven“ werden führende Krebsexperten die jüngsten wissenschaftlichen Erkenntnisse der Krebsmedizin sowie aktuelle gesundheitspolitische Aspekte der Krebsversorgung in Deutschland diskutieren.
\end{abstract}

\section{Essenziell: Die Sicht der Patienten}

„Durch die Wahl des Kongressmottos wollen wir auf die wissenschaftlichen und technologischen Fortschritte in der Onkologie hinweisen, die dafür sorgen, dass sich in der Krebsbehandlung neue Perspektiven eröffnen“, erklärt Prof. Thomas Wiegel, Kongresspräsident des DKK 2018. Als Beispiele nennt er die Weiterentwicklung der onkologischen Bildgebung, die mit dazu beiträgt, die moderne Präzisionsmedizin zu ermöglichen, sowie die Immuntherapie, die aktuell für Aufsehen sorgt. Doch trotz aller Fortschritte in der Krebsmedizin ist die Diagnose Krebs noch immer ein großer Einschnitt im Leben, der die Patienten vielfach zwingt, neue Lebensperspektiven zu entwickeln. Bei gutem Verlauf heißt das, mit der Krankheit leben zu lernen, im ungünstigen Fall ist der Krebs nicht mehr heilbar. „Ärzte sind dann gefragt, ihre Patienten so zu unterstützen, dass die verbleibende Lebenszeit zum einen noch möglichst lang, zum anderen aber auch möglichst beschwerdefrei ist. Dabei gilt es immer, den Patientenwunsch im Blick zu haben. Auch darüber wollen wir auf diesem Kongress diskutieren“, so Wiegel.

\section{Entscheidend: Interdisziplinärer Ansatz}

Der DKK 2018 zeichnet sich durch eine interdisziplinäre Besetzung aus, bei der alle Fachrichtungen vertreten sind, die an der Diagnose und Behandlung von Krebs mitwirken. In den Plenarsitzungen geht es unter anderem um die medizinische Entwick-

\section{vormerken: DKK 2018}

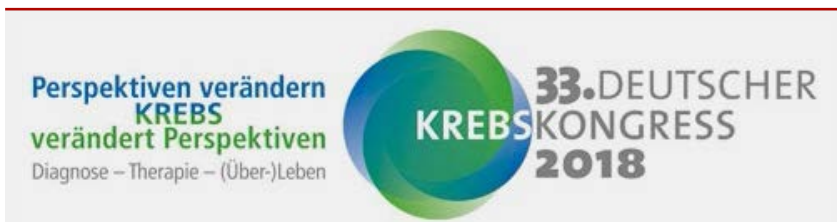

Wo: CityCube Berlin, Eingang Jafféstraße Wann: 21. bis 24. Februar 2018 Weitere Informationen, Vorprogramm und Anmeldung: www.dkk2018.de.
„Perspektiven verändern Krebs - Krebs verändert Perspektiven. Durch die Wahl dieses Kongressmottos wollen wir auf die wissenschaftlichen und technologischen Fortschritte in der Onkologie hinweisen, die dafür sorgen, dass sich in der Krebsbehandlung neue Perspektiven eröffnen."

Prof. Dr. Thomas Wiegel,

Präsident des Deutschen Krebskongresses 2018

lung bei ausgewählten Tumoren, um Neues aus Immuntherapie, Palliativmedizin und Psychoonkologie sowie um die Besonderheiten in der Behandlung von Metastasen. Dazu kommen interaktive Tumorkonferenzen und Sitzungen zu aktuellen onkologischen Leitlinien.

\section{Einprägsam: Neue Formate}

Ein besonderer Schwerpunkt liegt auf den sogenannten OxfordDebatten. Bei dieser besonderen Form der Pro- und-Kontra-Diskussion lernt das Publikum hoch komprimiert alle wichtigen Argumente hinter einer provokativen medizinischen Fragestellung kennen. In den gesundheitspolitischen Foren auf dem DKK 2018 sind unter anderem Diskussionen über die Weiterentwicklung der onkologischen Pflege sowie zur Finanzierbarkeit von Krebsarzneimitteln und Versorgungsstrukturen geplant. Der DKK 2018 bietet eine gute Gelegenheit, mit den gesundheits- und forschungspolitischen Vertretern der neuen Regierungskoalition über die Maßnahmen zu sprechen, die in der aktuellen Legislaturperiode zur Erreichung der Versorgungsziele des Nationalen Krebsplans nötig sind.

\section{Empathisch: Krebsaktionstag für Patienten und Angehörige}

Am 24. Februar öffnet der Kongress seine Tore für Patienten und Angehörige. Sie haben dann die Möglichkeit, sich in Vorträgen und im direkten Kontakt mit den Experten des Kongresses über die neuesten Entwicklungen der Krebsmedizin zu informieren, mit Selbsthilfegruppen Kontakt aufzunehmen und Krebsberatungsangebote kennenzulernen. In Podiumsdiskussionen und Interviews teilen Betroffene ihre Erfahrungen mit den Teilnehmern - eine gute Gelegenheit, miteinander ins Gespräch zu kommen. red. 